\title{
The Evaluation of the Clinical Effects of Botulinum Toxin on Nocturnal Bruxism
}

\author{
Fatih Asutay, ${ }^{1}$ Yusuf Atalay, ${ }^{2}$ Hilal Asutay, ${ }^{3}$ and Ahmet Hüseyin Acar ${ }^{4}$ \\ ${ }^{1}$ Faculty of Dentistry, Department of Oral and Maxillofacial Surgery, Afyon Kocatepe University, Afyonkarahisar, Turkey \\ ${ }^{2}$ Vefa Diş Polikliniği, Afyonkarahisar, Turkey \\ ${ }^{3}$ Uzman DentaClinic, Bursa, Turkey \\ ${ }^{4}$ Faculty of Dentistry, Department of Oral and Maxillofacial Surgery, Bezmialem Vakıf University, Istanbul, Turkey
}

Correspondence should be addressed to Fatih Asutay; dt_asutay@hotmail.com

Received 29 March 2017; Accepted 21 May 2017; Published 5 July 2017

Academic Editor: Lucindo Quintans

Copyright (c) 2017 Fatih Asutay et al. This is an open access article distributed under the Creative Commons Attribution License, which permits unrestricted use, distribution, and reproduction in any medium, provided the original work is properly cited.

\begin{abstract}
Objectives. Nocturnal bruxism can be managed by botulinum toxin (Botox ${ }^{\circledR}$ ) in patients who have not responded to conservative treatment. The aim of this study was to evaluate the efficacy of botulinum toxin A (BTXA) in the treatment of nocturnal bruxism. Material and Methods. The retrospective study comprised 25 female patients, aged 23-55 years (mean 35.84 \pm 8.41 years). All patients received a single injection of BTXA in the right and left masseters. Evaluation was made by Visual Analogue Scale (VAS) values, complaint duration, onset of effect, and duration of effectiveness. Results. BTXA produced significant improvements in pain scores. Only 2 adverse events (8\%) were recorded. Conclusion. BTX-A is effective in the treatment of nocturnal bruxism.
\end{abstract}

\section{Introduction}

Nocturnal bruxism (NB) is defined as abnormal maxillomandibular activity during sleep, characterized by grinding and clenching of the teeth $[1,2]$. NB can lead to wear on the teeth, dental prostheses/implant failure, tooth sensitivity, pain in the teeth, jaw, masticatory muscle, and temporomandibular joint (TMJ), neck pains and headache, periodontal disease, oral or facial pain, and potentially tooth loss $[3,4]$. These problems can be associated with the unconscious and intense contractions of the temporal and masseter muscles during sleep [5]. There are various treatment techniques for the management of NB such as oral splint, behavioral approaches, and medications (muscle relaxants, botulinum toxin) but none is widely accepted [4].

Botulinum toxin (Botox) is an exotoxin produced by the bacterium Clostridium botulinum, which blocks acetylcholine release from cholinergic nerve endings into the neuromuscular junction, thereby causing inactivity of muscles or glands [6]. Botulinum neurotoxin types A (BTXA) and B (BTXB) are approved by the United States Food and Drug Administration (FDA) including cervical dystonia, severe primary axillary hyperhidrosis, strabismus, blepharospasm, hemifacial spasm, and glabellar wrinkles for BTXA and cervical dystonia for BTXB.

BTXA has been used in the treatment of cosmetic and noncosmetic conditions such as tremor, hemifacial spasm, temporomandibular joint dysfunction, bruxism, masticatory myalgias, sialorrhea, hyperhidrosis, and headache [7].

Botox injections are directly applied into the masseter and temporalis muscles to relax these muscles. The clinical effects are typically seen on the first to third days after the injection, followed by one to two weeks of maximum effect, and the typical duration of the effect is three to four months [8].

In two comprehensive review studies, Tinastepe et al. [1] and Manfredini et al. [2] suggested that there is not enough evidence to use botulinum injection in the treatment of bruxism. The purpose of the present study was to investigate the potential performance of BTXA on nocturnal bruxism and to share this clinical experience.

\section{Materials and Methods}

In the period 2014-2015, a retrospective data analysis was made of the data of 25 female patients who underwent 
onabotulinum toxin A (Botox, Allergan, Inc., Irvine, CA, USA) injections into the masseter muscle for clinically diagnosed nocturnal bruxism. The patients enrolled in the study had all failed to respond to conservative treatment. Exclusion criteria were temporomandibular disorders, pregnancy, active drug use, and psychological therapy.

For evaluation of postoperative pain, Visual Analogue Scale (VAS) forms were completed by the patients scoring the degree of pain between 0 (absence of pain) and 10 (maximum pain) before the injection and then at 2 weeks, 1 month, 3 months, 4 months, and 6 months after the injection.

The following data were recorded for all patients: maximum mouth opening changes, duration of the complaint before the injection, muscle weakness before injection, the time the first effects were seen, the time when the effectiveness started to be lost, and difficulty experienced in speaking, swallowing, or chewing.

This study was based on routine service documents of NB patients. However, permission to analyse the data was sought from the management of the Dentistry Hospital of Afyon Kocatepe University. All procedures followed were in accordance with the ethical standards of the committee responsible for human experimentation (institutional and national) and with the Helsinki Declaration of 1975, as revised in 2008. Written informed consent was obtained from all the study participants.

2.1. Procedure. For all patients, 100 mouse units (MU) of onabotulinum toxin A (Botox, Allergan, Inc., Irvine, CA) were diluted in $2 \mathrm{ml}$ of saline.

In all patients, a dose of $20 \mathrm{MU}$ of BTXA was injected into a single masseter muscle using a $0.5 \mathrm{~mL}$ insulin syringe at four points $(5 \mathrm{MU} / \mathrm{site})$. The patient was requested to clench the masseter muscle, and the injections were applied to the origin, insertion, anterior, and posterior parts of the muscle.

2.2. Statistical Analysis. Statistical analysis was performed with the Statistical Package for the Social Science Program (version 20.0, SPSS Inc., Chicago, USA). Differences in individual parameters were tested using the repeated measures test ANOVA and post hoc Friedman and Wilcoxon test for abnormally distributed variables (pain). A value of $p<0.05$ was considered statistically significant.

\section{Results}

Evaluation was made of the data of 25 female patients with a mean age of $35.84 \pm 8.41$ years (range, $23-55$ years). All the study variables are shown in Tables 1 and 2.

One patient had pain in the injection points. No adverse effects or dry mouth (injection into parotid gland) was reported in any other patients.

There were no significant changes in respect of the maximum mouth opening. Only 2 patients (8\%) had no significant improvements in the pain scores after treatment.

The mean duration of the complaint before injection was $8.84 \pm 4.57$ years, the time that the effects were first seen was
$12.24 \pm 2.02$ days, and the time that the loss of effectiveness started was $4.76 \pm 1.01$ months (Table 3 ).

The differences between two-week-four-month and onemonth-three-month postoperative pain values were not statistically significant. However, differences between all other times were statistically significant (Table 4 ).

\section{Discussion}

In the present study, there were significant differences in reduction of pain and bruxism activity.

Van Zandijcke and Marchau [9] used botulinum toxin for the first time in the treatment of bruxism of a young woman with a brain injury, and a marked reduction was observed. Thereafter, botulinum toxin was used for the treatment of bruxism with different origins such as cranial-cervical dystonia [10], Huntington's disease [11], autism [12], and amphetamine addiction [13].

To date, only BTXA and BTXB serotypes have been approved by the US Food and Drug Administration (FDA) for clinical use including cervical dystonia, severe primary axillary hyperhidrosis, strabismus, blepharospasm, hemifacial spasm, and glabellar wrinkles for BTXA and cervical dystonia for BTXB.

Lang [14] reported that BTXA showed better and longer pain relief than BTXB. Moreover, BTXA had fewer sideeffects than BTXB [14]. In our clinic, BTXA has been safely used for the treatment of bruxism, masseteric hypertrophy, and sialorrhea.

Although the pathogenesis of bruxism remains unclear, it is generally accepted that the etiology is multifactorial in nature. The occurrence of nocturnal bruxism suggests that it may occur as a result of possible physical or psychological conditions (emotional stress, anxiety, aggressive and hyperactive personality types, malocclusion, sleep problems such as sleep apnea, earache, headache, and tooth ache, the sideeffects of some psychiatric medications, etc.) $[13,15]$.

The treatment of myofascial pain can be difficult for both clinicians and patients because most of these disorders involve multiple components such as somatic, neurogenic, and psychogenic components. Moreover, it can be resistant to conventional medical or behavioral therapy. There is no consensus on how the effects of Botox reduce pain. Some hypotheses have suggested changes in levels of muscle nociceptor sensitizers, nonacetylcholine neurotransmitters, and acetylcholine at autonomic synapses [16-18] and decreased motor neuron activity [19].

Nocturnal bruxism may lead to pain in the head, neck, jaw, teeth, and temporomandibular joint (TMJ). However, conservative treatments may be limited in the resolution of this problem. In the past few years, the use of Botox therapy has become a promising source for the management of myofascial pain [20]. The findings related to pain in the current study showed a significant difference before and after the injection, which is consistent with the findings of Sidebottom AJ et al. [21], Guarda-Nardini et al. [22], and Tan et al. [23].

In a study by Lee et al. [24], it was reported that BTXA injection reduced the number of bruxism events during sleep 
TABLE 1: The demographic data, pain scores, the time of the onset of the effect, the time of the onset of the loss of effect, and the duration of the complaint of all the patients.

\begin{tabular}{|c|c|c|c|c|c|c|c|c|c|c|}
\hline \multirow{2}{*}{ Patient } & \multirow{2}{*}{ Age } & \multicolumn{6}{|c|}{ VAS Scores } & \multirow{2}{*}{ Onset of effect (day) } & \multirow{2}{*}{ Onset of lose of effect (month) } & \multirow{2}{*}{ Duration of complaint (year) } \\
\hline & & Pre-op & 2.w & $1 . \mathrm{m}$ & $3 . \mathrm{m}$ & $4 . \mathrm{m}$ & $6 . \mathrm{m}$ & & & \\
\hline 1 & 25 & 8 & 1 & 1 & 0 & 2 & 6 & 10 & 4 & 1 \\
\hline 2 & 23 & 5 & 1 & 1 & 3 & 4 & 5 & 13 & 3 & 4 \\
\hline 3 & 24 & 7 & 5 & 1 & 1 & 1 & 7 & 14 & 6 & 1 \\
\hline 4 & 29 & 8 & 3 & 1 & 0 & 0 & 3 & 14 & 5 & 8 \\
\hline 5 & 55 & 8 & 7 & 6 & 6 & 7 & 8 & 15 & 4 & 12 \\
\hline 6 & 36 & 6 & 2 & 1 & 1 & 1 & 2 & 12 & 5,5 & 5 \\
\hline 7 & 31 & 7 & 0 & 0 & 0 & 1 & 3 & 10 & 5 & 15 \\
\hline 8 & 26 & 8 & 2 & 4 & 7 & 7 & 7 & 15 & 2,5 & 9 \\
\hline 9 & 40 & 9 & 5 & 2 & 2 & 2 & 4 & 13 & 5 & 11 \\
\hline 10 & 41 & 6 & 3 & 1 & 1 & 1 & 4 & 12 & 5 & 7 \\
\hline 11 & 36 & 7 & 3 & 0 & 0 & 2 & 4 & 11 & 6 & 9 \\
\hline 12 & 34 & 4 & 0 & 0 & 0 & 0 & 1 & 10 & 5 & 4 \\
\hline 13 & 37 & 6 & 2 & 1 & 1 & 1 & 2 & 14 & 5 & 5 \\
\hline 14 & 29 & 7 & 2 & 1 & 1 & 2 & 3 & 12 & 4,5 & 8 \\
\hline 15 & 48 & 7 & 1 & 0 & 0 & 0 & 1 & 13 & 5,5 & 10 \\
\hline 16 & 43 & 6 & 3 & 2 & 2 & 2 & 3 & 15 & 5 & 3 \\
\hline 17 & 51 & 8 & 1 & 0 & 0 & 2 & 3 & 9 & 4 & 12 \\
\hline 18 & 46 & 8 & 3 & 1 & 1 & 1 & 2 & 9 & 4 & 14 \\
\hline 19 & 33 & 9 & 4 & 1 & 1 & 1 & 3 & 11 & 5 & 18 \\
\hline 20 & 32 & 7 & 0 & 0 & 0 & 1 & 2 & 8 & 4 & 13 \\
\hline 21 & 37 & 7 & 2 & 0 & 0 & 1 & 2 & 14 & 4 & 7 \\
\hline 22 & 29 & 9 & 2 & 1 & 1 & 1 & 1 & 13 & 6 & 6 \\
\hline 23 & 42 & 8 & 7 & 7 & 7 & 7 & 7 & 13 & 5 & 15 \\
\hline 24 & 35 & 6 & 0 & 0 & 0 & 0 & 1 & 12 & 5,5 & 13 \\
\hline 25 & 34 & 7 & 1 & 0 & 0 & 0 & 1 & 14 & 5 & 11 \\
\hline
\end{tabular}

Abbreviations. VAS: Visual Analogue Scale, BTXA: Botulinum Toxin A, w: week, m: month.

TABLE 2: The characteristics of the patients according to the pain scores.

\begin{tabular}{lc}
\hline & VAS \\
\hline Before injection & $7.12 \pm 1.236(4-9)$ \\
2nd week & $2.40 \pm 1.979(0-7)$ \\
1st month & $1.28 \pm 1.815(0-7)$ \\
3rd month & $1.40 \pm 2.141(0-7)$ \\
4th month & $1.88 \pm 2.128(0-7)$ \\
6th month & $3.40 \pm 2.141(1-8)$ \\
\hline
\end{tabular}

and it was therefore suggested that BTXA injection could be used as an effective treatment for nocturnal bruxism. Similarly, Santamato et al. [25] reported that neck pain related to nocturnal bruxism can be treated with BTXA. In the study by Santamato et al. [25], the dose of BTXA injected into each masseter muscle was $40 \mathrm{MU}$ and $25 \mathrm{MU}$ was injected into each temporalis muscle. Moreover, electromyography was used to measure to the changes of masseter and temporalis muscle hyperactivities and VAS was used to measure to pain
[25]. In the present study, $20 \mathrm{MU}$ BTXA was injected into each masseter muscle and positive results were reported.

Kesikburun et al. [26] reported a 21-year-old male patient with traumatic brain injury, who was successfully treated with BTXA for bruxism. According to our clinical experience, BTXA can be used safely for the treatment of bruxism.

The maximum mouth opening may be increased after injection of BTXA. Sidebottom et al. [21] and Guarda-Nardini et al. [22] reported that the interincisal distance can be induced with BTXA injection. However, the results of the current study do not match this conclusion. The differences may be due to most of the patients having no previous complaint of limited mouth opening or they may have been due to the small sample size.

The limitations of the present study include the small sample size and that there was no information about temporal injection or difference between genders.

In conclusion, Botox therapy seems promising and beneficial in the treatment of nocturnal bruxism, although several limiting factors such as high cost and the necessity for repeated injections prevent its widespread use. When there has been no response to conservative treatment methods, 
TABLE 3: The characteristics of the patients according to age, onset of effect, onset of loss of effect, and duration of complaint.

\begin{tabular}{lcccc}
\hline & Age & Onset of effect (day) & Onset of loss of effect (month) & Duration of complaint (year) \\
\hline Mean \pm SD & $35.84 \pm 8.41$ & $12.24 \pm 2.02$ & $4.76 \pm 1.01$ & $8.84 \pm 4.57$ \\
Min-max & $23-55$ & $8-15$ & $2-6$ & $1-18$ \\
\hline
\end{tabular}

TABLE 4: Test statistics ${ }^{\mathrm{a}} .{ }^{\mathrm{a}}$ Wilcoxon signed ranks test. ${ }^{\mathrm{b}}$ Based on positive ranks. ${ }^{\mathrm{c}}$ Based on negative ranks.

\begin{tabular}{|c|c|c|c|c|c|}
\hline & vas $2 \mathrm{w}$-vaspi & Vaslm-vaspi & Vas3m-vaspi & Vas4m-vaspi & Vas6m-vaspi \\
\hline$Z$ & $-4,390^{\mathrm{b}}$ & $-4,410^{\mathrm{b}}$ & $-4,395^{\mathrm{b}}$ & $-4,392^{\mathrm{b}}$ & $-4,124^{\mathrm{b}}$ \\
\hline \multirow[t]{2}{*}{ Asymp.Sig. (2-tailed) } &, 000 & 000 &, 000 &, 000 &, 000 \\
\hline & vas1m-vas $2 \mathrm{w}$ & vas $3 \mathrm{~m}-\mathrm{vas} 2 \mathrm{w}$ & Vas $4 \mathrm{~m}$-vas $2 \mathrm{w}$ & vas $6 m$-vas $2 \mathrm{w}$ & \\
\hline$Z$ & $-3,257^{\mathrm{b}}$ & $-2,751^{\mathrm{b}}$ & $-1,629^{b}$ & $-2,644^{\mathrm{c}}$ & \\
\hline \multirow[t]{2}{*}{ Asymp.Sig. (2-tailed) } & ,001 &, 006 & ,103 &, 008 & \\
\hline & vas3m-vas1m & vas $4 \mathrm{~m}$-vas1m & vas6m-vas1m & vas $4 m$-vas $3 m$ & \\
\hline$Z$ &,$- 736^{\mathrm{c}}$ & $-2,656^{c}$ & $-4,234^{\mathrm{c}}$ & $-2,762^{c}$ & \\
\hline \multirow[t]{2}{*}{ Asymp.Sig. (2-tailed) } & ,461 & ,008 &, 000 & ,006 & \\
\hline & vas $6 \mathrm{~m}$-vas $3 \mathrm{~m}$ & Vas6m-vas $4 \mathrm{~m}$ & & & \\
\hline$Z$ & $-4,148^{\mathrm{c}}$ & $-4,239^{c}$ & & & \\
\hline Asymp.Sig. (2-tailed) &, 000 &, 000 & & & \\
\hline
\end{tabular}

vaspi: VAS score at preinjection; vas2w: VAS score at 2 weeks (after injection); vas1m: VAS score in first month; vas3m: VAS score in third month; vas4m: VAS score in fourth month; vas6m: VAS score in sixth month.

botulinum toxin may be an alternative and effective treatment for nocturnal bruxism and masticatory pain. Future studies with a larger number of patients are required to confirm the conclusions reached in the present study.

\section{Consent}

Patient consent was obtained.

\section{Conflicts of Interest}

The authors declare that they have no conflicts of interest.

\section{Acknowledgments}

Special thanks are due to Professor İsmet Doğan for his support in the statistical analyses.

\section{References}

[1] N. Tinastepe, B. B. Küçük, and K. Oral, "Botulinum toxin for the treatment of bruxism," Cranio, vol. 33, no. 4, pp. 292-299, 2015.

[2] D. Manfredini, J. Ahlberg, E. Winocur, and F. Lobbezoo, "Management of sleep bruxism in adults: a qualitative systematic literature review," Journal of Oral Rehabilitation, vol. 42, no. 11, pp. 862-874, 2015.

[3] M. C. Carra, N. Huynh, and G. Lavigne, "Sleep bruxism: a comprehensive overview for the dental clinician interested in sleep medicine," Dental Clinics of North America, vol. 56, no. 2, pp. 387-413, 2012.
[4] K. Koyano, Y. Tsukiyama, R. Ichiki, and T. Kuwata, "Assessment of bruxism in the clinic," Journal of Oral Rehabilitation, vol. 35, no. 7, pp. 495-508, 2008.

[5] Y. J. Shim, M. K. Lee, T. Kato, H. U. Park, K. Heo, and S. T. Kim, "Effects of botulinum toxin on jaw motor events during sleep in sleep bruxism patients: a polysomnographic evaluation," Journal of Clinical Sleep Medicine, vol. 10, no. 3, pp. 291-298, 2014.

[6] L. C. Sellin and S. Thesleff, "Pre- and post-synaptic actions of botulinum toxin at the rat neuromuscular junction.," The Journal of Physiology, vol. 317, no. 1, pp. 487-495, 1981.

[7] A. Blitzer and L. Sulica, "Botulinum toxin: basic science and clinical uses in otolaryngology," Laryngoscope, vol. 111, no. 2, pp. 218-226, 2001.

[8] G. E. Borodic, M. Acquadro, and E. A. Johnson, "Botulinum toxin therapy for pain and inflammatory disorders: mechanisms and therapeutic effects," Expert Opinion on Investigational Drugs, vol. 10, no. 8, pp. 1531-1544, 2001.

[9] M. Van Zandijcke and M. M. Marchau, "Treatment of bruxism with botulinum toxin injections," Journal of Neurology Neurosurgery and Psychiatry, vol. 53, no. 6, p. 530, 1990.

[10] M. W. Watts, E.-K. Tan, and J. Jankovic, "Bruxism and cranialcervical dystonia: is there a relationship?” Cranio, vol. 17, no. 3, pp. 196-201, 1999.

[11] M. C. Nash, R. B. Ferrell, M. A. Lombardo, and R. B. Williams, "Treatment of bruxism in Huntington's disease with botulinum toxin [2]," Journal of Neuropsychiatry and Clinical Neurosciences, vol. 16, no. 3, pp. 381-382, 2004.

[12] P. G. Monroy and M. A. Da Fonseca, "The use of botulinum toxin-A in the treatment of severe bruxism in a patient with autism: a case report," Special Care in Dentistry, vol. 26, no. 1, pp. 37-39, 2006. 
[13] S.-J. See and E.-K. Tan, "Severe amphethamine-induced bruxism: treatment with botulinum toxin," Acta Neurologica Scandinavica, vol. 107, no. 2, pp. 161-163, 2003.

[14] A. M. Lang, "A preliminary comparison of the efficacy and tolerability of botulinum toxin serotypes $\mathrm{A}$ and $\mathrm{B}$ in the treatment of myofascial pain syndrome: a retrospective, openlabel chart review," Clinical Therapeutics, vol. 25, no. 8, pp. 22682278, 2003.

[15] G. D. Klasser, N. Rei, and G. J. Lavigne, "Sleep bruxism etiology: the evolution of a changing paradigm," Journal of the Canadian Dental Association, vol. 81, no. f2, 2015.

[16] A. G. Hohmann and M. Herkenham, "Cannabinoid receptors undergo axonal flow in sensory nerves," Neuroscience, vol. 92, no. 4, pp. 1171-1175, 1999.

[17] A. Bengtsson, K. G. Henriksson, and J. Larsson, "Reduced high-energy phosphate levels in the painful muscles of patients with primary fibromyalgia," Arthritis \& Rheumatism, vol. 29, no. 7, pp. 817-821, 1986.

[18] A. H. Wheeler, "Myofascial pain disorders: theory to therapy," Drugs, vol. 64, no. 1, pp. 45-62, 2004.

[19] C. C. Donaldson, D. V. Nelson, and R. Schulz, "Disinhibition in the gamma motoneuron circuitry: a neglected mechanism for understanding myofascial pain syndromes?" Applied Psychophysiology Biofeedback, vol. 23, no. 1, pp. 43-59, 1998.

[20] C. Kurtoglu, O. H. Gur, M. Kurkcu, Y. Sertdemir, F. Guler-Uysal, and $\mathrm{H}$. Uysal, "Effect of botulinum toxin-A in myofascial pain patients with or without functional disc displacement," Journal of Oral and Maxillofacial Surgery, vol. 66, no. 8, pp. 1644-1651, 2008.

[21] A. J. Sidebottom, A. A. Patel, and J. Amin, "Botulinum injection for the management of myofascial pain in the masticatory muscles: a prospective outcome study," British Journal of Oral and Maxillofacial Surgery, vol. 51, no. 3, pp. 199-205, 2013.

[22] L. Guarda-Nardini, D. Manfredini, M. Salamone, L. Salmaso, S. Tonello, and G. Ferronato, "Efficacy of botulinum toxin in treating myofascial pain in bruxers: a controlled placebo pilot study," Cranio, vol. 26, no. 2, pp. 126-135, 2008.

[23] E.-K. Tan and J. Jankovic, "Treating severe bruxism with botulinum toxin," Journal of the American Dental Association, vol. 131, no. 2, pp. 211-216, 2000.

[24] S. J. Lee, W. D. McCall, Y. K. Kim, S. C. Chung, and J. W. Chung, "Effect of botulinum toxin injection on nocturnal bruxism: a randomized controlled trial," American Journal of Physical Medicine and Rehabilitation, vol. 89, no. 1, pp. 16-23, 2010.

[25] A. Santamato, F. Panza, D. Di Venere et al., "Effectiveness of botulinum toxin type A treatment of neck pain related to nocturnal bruxism: a case report," Journal of Chiropractic Medicine, vol. 9, no. 3, pp. 132-137, 2010.

[26] S. Kesikburun, R. Alaca, B. Aras, I. Tuğcu, and A. K. Tan, "Botulinum toxin injection for bruxism associated with brain injury: case report," Journal of Rehabilitation Research and Development, vol. 51, no. 4, pp. 661-664, 2014. 


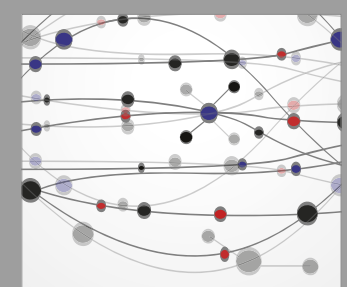

The Scientific World Journal
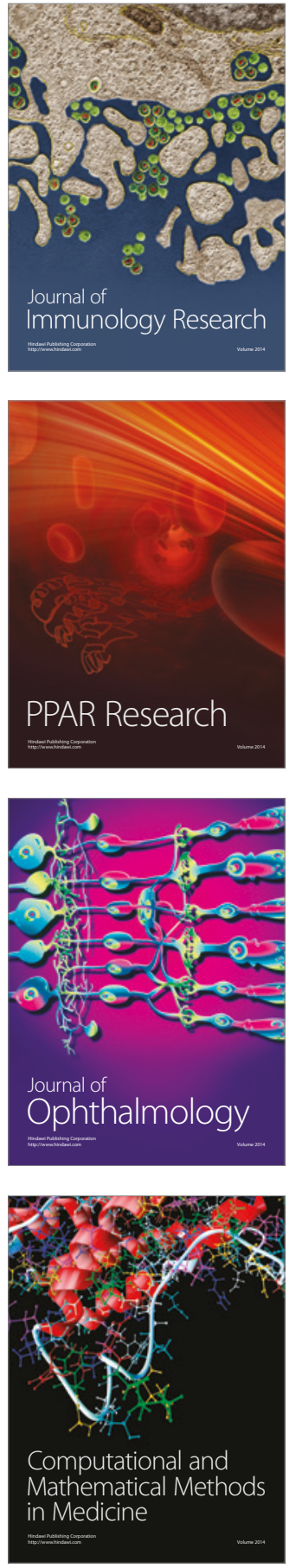

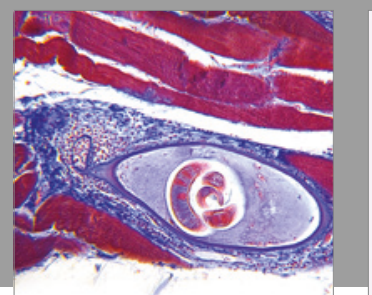

Gastroenterology Research and Practice
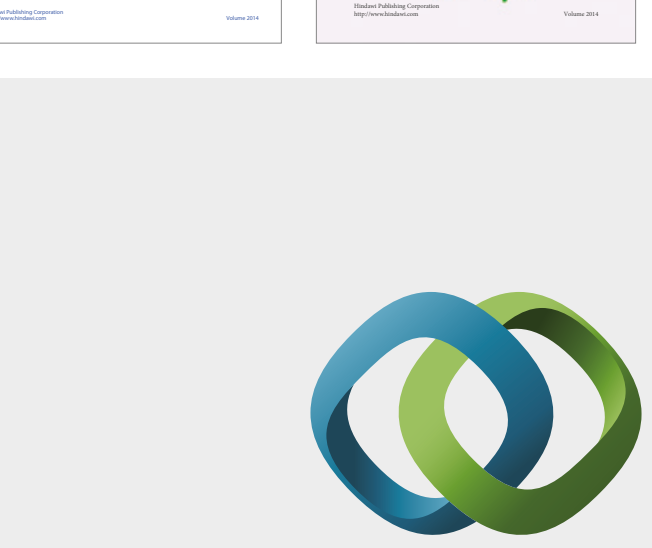

\section{Hindawi}

Submit your manuscripts at

https://www.hindawi.com
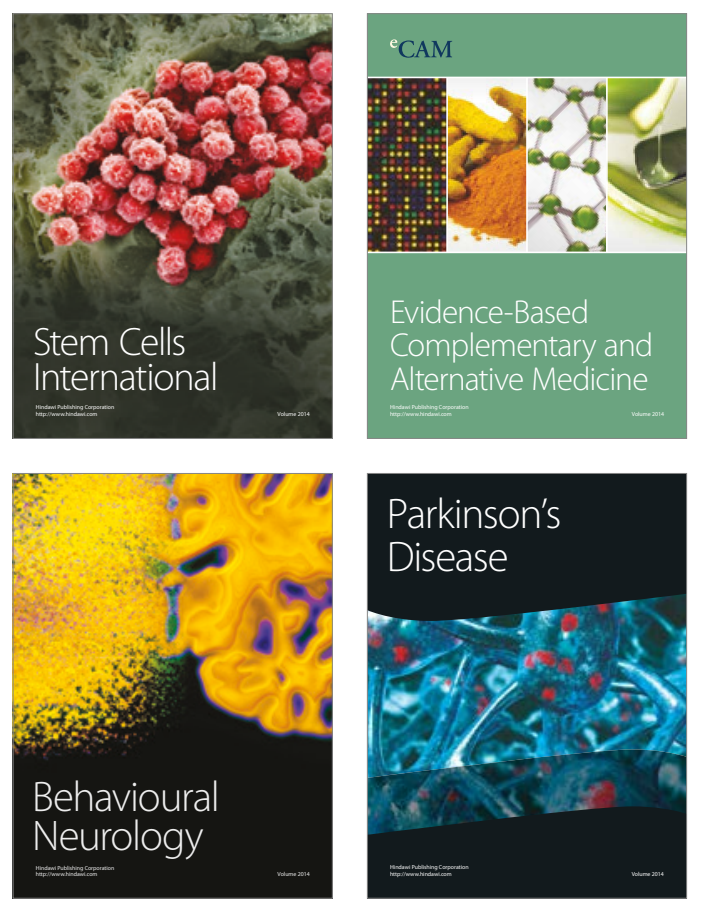
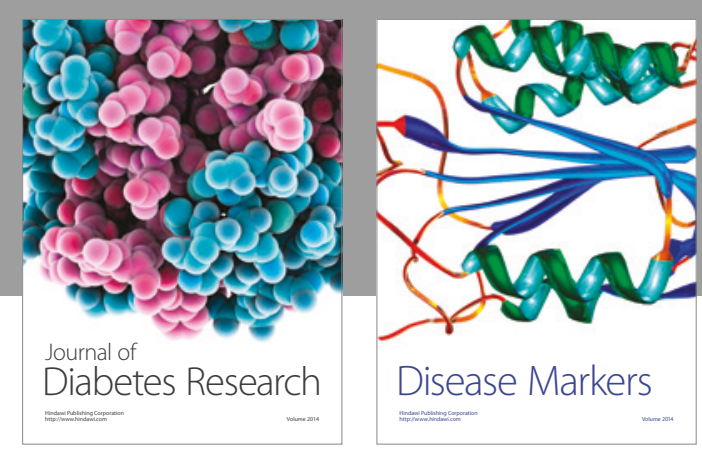

Disease Markers
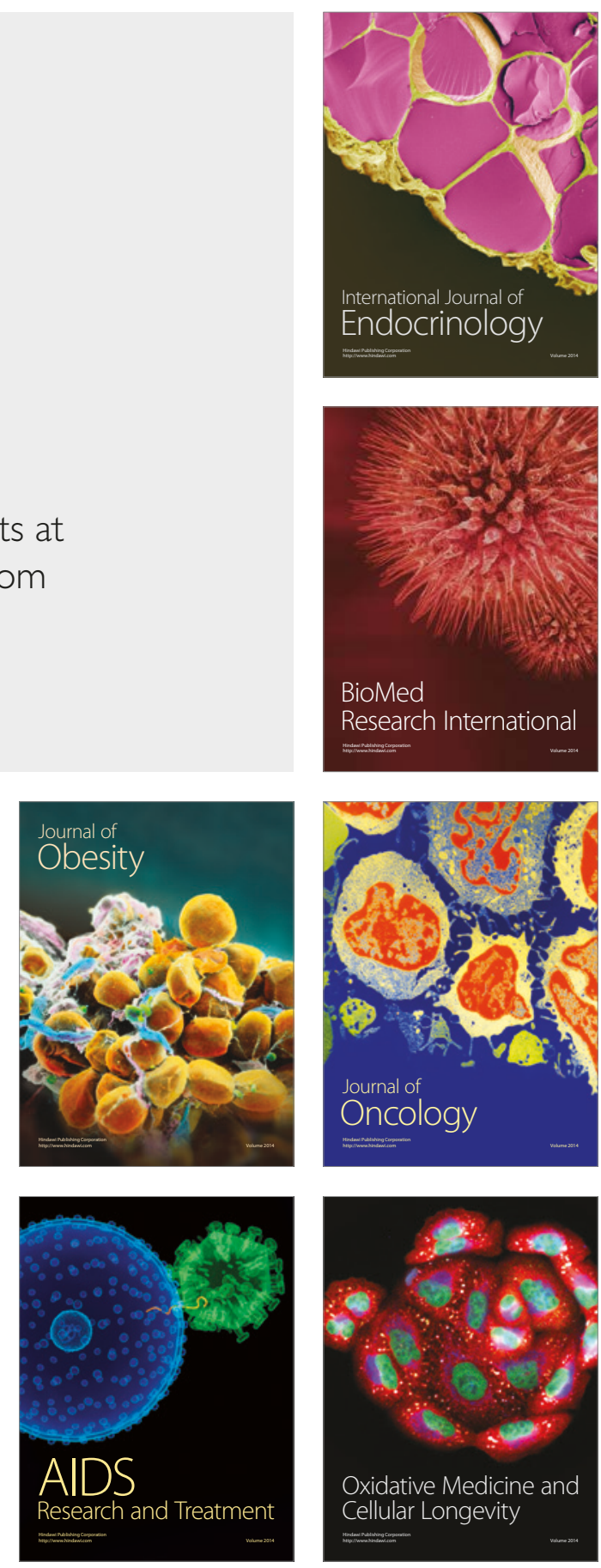